\title{
El webcast como estrategia de aprendizaje significativo en la Fisiología Cardiovascular
}

\author{
The webcast as a Meaningful Learning Strategy in Cardiovascular \\ Physiology
}

\section{Webcast como uma estratégia de aprendizagem significativa em Fisiologia Cardiovascular}

Hernan Dario Delgado-Rico, MD., MSc. *

Hilda Leonor Gonzalez-Olaya, MD., MSc. **

\section{Resumen}

Introducción: En la actualidad, se hace necesario transformar las actividades educativas para incluir a los "nativos digitales" quienes actualmente componen la mayoría de los estudiantes en las aulas. En el presente estudio se aborda el resultado de una investigación educativa en Ciencias de la Salud usando una metodología de innovación basada en la incorporación de Tecnologías de Información y Comunicación, el webcast. Objetivo: Evaluar el impacto en el aprendizaje de temas de Fisiología cardiorrespiratoria mediante un webcast aplicado en estudiantes de Medicina de segundo año de una universidad colombiana Metodología: Se realizó un estudio cuasiexperimental, que incluyó estudiantes matriculados en segundo año de medicina de una universidad del nororiente colombiano. Para evaluar el impacto en el aprendizaje, se utilizó una prueba estandarizada de conocimentos sobre Fisiología respiratoria y cardiovascular. Resultados: Se encontró mejor desempeño en el grupo que se instruyó usando webcast comparado con el de las clases magistrales $(\bar{x}=3.47 \pm 0.04$ versus $3.18 \pm 0.03$, $\pm E E M$, respectivamente; ANOVA, $F=6.56, p=0.01$ ). Además, se aplicó una encuesta sobre aspectos del uso y opiniones sobre los materiales. Conclusiones: El webcast es una metodología que permite reorganizar las actividades de formación potenciando procesos de pensamiento formal y logra el aprendizaje significativo de tópicos relacionados con la fisiología cardiovascular; adicionalmente, en los estudiantes se encontró buena recepción de la estrategia innovadora empleada y les permitió tener un desempeño satisfactorio en las pruebas de evaluación de conocimientos, considerándolo como un buen complemento de currículos que lo combinen junto a las disertaciones magistrales. [Delgado-Rico HD, Gonzalez-Olaya HL. El webcast como estrategia de aprendizaje significativo en la Fisiología Cardiovascular. MedUNAB 2017; 20(2): 123-130].

Palabras clave: Difusión por la Web; Educación Médica; Desarrollo Tecnológico; Fisiología; Aprendizaje.

* Médico, Universidad Industrial de Santander, magíster en Ciencias Biomédicas, Universidad Industrial de Santander, magíster en Educación, Instituto Tecnológico de Monterrey. Profesor Área de Ciencias Médicas Básicas, UniversidadAutónoma de Bucaramanga(UNAB), Santander, Bucaramanga, Colombia. ** Médica, Universidad Industrial de Santander, magíster en Neurociencias, Universidad Internacional de Andalucía, magíster en Fisiología, Universidad de Toronto. Profesor Área de Ciencias Médicas Básicas, UniversidadAutónoma de Bucaramanga(UNAB), Santander, Bucaramanga, Colombia.

Correspondencia: Hernan Dario Delgado Rico. Calle 157 No. 19-55, Cañaveral Parque, Floridablanca, Santander Colombia. Teléfono: +57 (7) 6436111 ext. 551.E-mail:hdelgado3@unab.edu.co 


\section{Abstract}

Introduction: Nowadays, it is necessary to transform educational activities to include the "digital natives" who currently make up the vast majority of students in the classrooms. This study is the result of an educational research in Health Sciences which is addressed using an innovation methodology based on the incorporation of Information and Communication Technologies, the webcast. Objective: To evaluate the impact on learning of Cardiorespiratory Physiology topics making use of a webcast applied to second-year medicine students of a Colombian university. Methodology: A quasi-experimental study was conducted, which included students who are enrolled in the second year of medicine at a university located in the northeast of Colombia. A standardized knowledge test on respiratory and cardiovascular physiology was used to evaluate the impact on learning. Results: The group instructed using webcast had a better performance in the knowledge test compared to group instructed in master classes $(\bar{x}=3.47 \pm 0.04$ versus $3.18 \pm 0.03, \pm E E M$ respectively; ANOVA, $F=6.56, p=0.01$ ). Additionally, a survey was applied regarding aspects of the use and opinions of the materials. Conclusions: A webcast is a methodology that allows the reorganization of training activities which can promote formal thought processes, and achieve the meaningful learning of topics related to cardiovascular physiology. In addition, a good reception of the innovative strategy used was found in students. Also, it allowed them to have a satisfactory performance in knowledge tests which is considered as a good complement to curricula if it is combined it with master classes. [Delgado-Rico HD, Gonzalez-Olaya HL. The webcast as a Meaningful Learning Strategy in Cardiovascular Physiology. MedUNAB 2017; 20(2): 123-130].

Keywords: Webcasts; Education, Medical; Technological Development; Physiology; Learning.

\section{Introducción}

La Educación tiene como propósito la transformación de la sociedad hacia objetivos nobles y uno de ellos es facilitar los procesos de enseñanza-aprendizaje en procura de incluir los diferentes estilos de aprendizaje de las personas y potenciar sus capacidades de resolver las situaciones a las que se enfrenta. Las Tecnologías de Información y Comunicación (TIC) permiten apoyar la democratización del acceso a la información a la vez que brindan flexibilidad y calidad siempre y cuando lleve un proceso juicioso de construcción de los recursos educativos, los cuales idealmente deberían ser abiertos. En conjunto, la Educación y las TIC tienen amplias y diversas opotunidades de complementarse dentro de los entornos educativos, adaptándolos a las necesidades de cada ámbito profesional. En el campo de Ciencias de la Salud, existen necesidades importantes de flexibilizar los programas de instrucción para poder llegar a lugares distantes, solucionar necesidades de capacitación en

\section{Resumo}

Introdução: No momento, é necessário transformar atividades educacionais para incluir os "nativos digitais" que atualmente compõem a maioria dos alunos nas salas de aula. No presente estudo, o resultado de uma pesquisa educacional em Ciências da Saúde é abordado usando uma metodologia de inovação baseada na incorporação de Tecnologias de Informação e Comunicação, o webcast. Objetivo: Avaliar o impacto na aprendizagem dos temas da Fisiologia Cardiorrespiratória através de uma transmissão na webcast, aplicada a estudantes de medicina de segundo ano de uma universidade colombiana. Metodologia: Foi realizado um estudo quase-experimental, que incluiu estudantes matriculados no segundo ano de medicina numa universidade no nordeste da Colômbia. Para avaliar o impacto na aprendizagem, foi utilizado um teste de conhecimento padronizado em fisiologia respiratória e cardiovascular. Resultados: Encontramos um melhor desempenho no grupo que foi instruído a usar webcast em comparação com as classes magistrais $(\bar{x}=3.47 \pm 0.04$ contra $3.18 \pm 0.03, \pm E E M$, respetivamente, ANOVA, $F=6.56, p=0.01$ ). Além disso, foi aplicada uma pesquisa sobre aspetos do uso e opiniões sobre os materiais. Conclusões: O webcast é uma metodologia que permite reorganizar as atividades de treinamento fortalecendo os processos de pensamento formal e a aprendizagem significativa de tópicos relacionados à fisiologia cardiovascular; Além disso, os alunos encontraram uma boa aceitação da estratégia inovadora utilizada e permitiram que eles tivessem um desempenho satisfatório nos testes de avaliação do conhecimento, considerando-o como um bom complemento aos currículos que o combinam com as aulas magistrais. [Delgado-Rico HD, Gonzalez-Olaya HL. Webcast como uma estratégia de aprendizagem significativa em Fisiologia Cardiovascular. MedUNAB 2017; 20(2): 123-130].

Palavras-chave: Webcasts; Educação Médica; Desenvolvimento Tecnológico; Fisiologia; Aprendizagem.

horarios poco convencionales y hasta el momento apenas existen estudios incipientes que aborden las consecuencias de aplicar innovaciones educativas en la formación de profesionales de esta rama.

Gracias a la incorporación de las TIC en la educación ha sido posible crear alternativas didácticas aplicables en diversos niveles de entrenamiento y han permitido fortalecer los modelos de enseñanza-aprendizaje que están centrados en los aprendices; asimismo, han permitido franquear las barreras de espacio y tiempo en la instrucción a la vez que se propende por buscar nuevas formas de aprender a través de diferentes técnicas y herramientas que se desarrollan en ambientes flexibles, adaptativos y abiertos a los diferentes rasgos cognitivos de los estudiantes estimulando su autonomía y el desarrollo de competencias (1-3). Como consecuencia esperada de estas innovaciones, es necesaria la evaluación del impacto en el aprendizaje de estas estrategias novedosas con la finalidad de hacer ajustes a las mismas y extraer sus mejores aportes. 
En el ámbito de Ciencias de la Salud, estas aplicaciones tecnológicas se han incorporado como importante componente del actuar de los profesionales y se hace necesario anexarlas en la educación desde niveles de pregrado profesional y formación técnica hasta el postgrado, así como a los programas de formación continuada. Gracias al uso de TIC en la Educación de Ciencias de la Salud ha ocurrido una transformación progresiva desde el esquema tradicional fundamentado en disertaciones magistrales hacia un modelo modernizado en donde se pretende lograr las competencias tecnológicas junto a las disciplinares en los aspectos científicos, epidemiológicos, clínicos y de comunicación con los miembros del equipo interdisciplinario, el paciente y sus familiares $(2,4)$.

Debido al uso cada vez más generalizado de las TIC, se ha podido franquear en alto grado la dificultad para acceder a información de toda índole en Ciencias de la Salud, pero a su vez esto ha creado nuevos derroteros para el proceso de enseñanza aprendizaje porque es necesario que los aprendices desarrollen habilidades nuevas con relación a todo este gran cúmulo de información disponible. Se desea que los estudiantes seleccionen las informaciones con mayor solidez de fuente y las procesen en un tiempo óptimo que vaya acorde a la exigencia que implica tomar decisiones prontas para evitar complicaciones con los pacientes, en el marco de un agudo sentido crítico que permita decidir qué aprender, cómo aprenderlo y el lugar indicado para hacerlo; en conjunto, desarrollar estas competencias le permitirá al futuro profesional resolver de forma adecuada los problemas que se le presentarán en su ejercicio, igualmente, gestionar adecuadamente su conocimiento y actualización dentro de la continua y abundante producción de conocimiento científico $(5,6)$.

De esta forma, han aparecido videos instruccionales que han tenido aceptación importante en los estudiantes universitarios, por ejemplo, la Academia Khan que actualmente alberga cientos de estos videos en ramas diversas de la Ciencia. Algunas revistas de impacto renombrado en Ciencias de la Salud, como el "New England Journal of Medicine" o la revista JAMA, utilizan estas herramientas multimedia para difundir contenidos de sus publicaciones $(7,8)$. Más recientemente, diversas universidades e instituciones de salud han creado canales en YouTube o plataformas similares, para entregar videos científicos dirigidos a estudiantes e incluso a pacientes; por ejemplo, el Hospital John Hopkins de Estados Unidos cuenta con más de 32,000 suscriptores y más de 8 millones de reproducciones en su canal de Youtube(9).

Las TIC han posibilitado el desarrollo de contenidos generados por los usuarios a través de aplicaciones conocidas como web 2.0, ejemplos de ello son los vodcast y podcast que son archivos de video y audio diseñados especialmente para descargarse a dispositivos móviles como tabletas y teléfonos celulares. Las ventajas de estos productos se cuentan en su portabilidad, facilidad y asequibilidad de acceso y uso, e incluso, la posibilidad de dar libertad al usuario para hacer derivaciones de estos materiales, este escenario ha sido descrito con el concepto de "educación móvil"(10) y actualmente hay notorio interés de muchas instituciones de formación en Ciencias de la Salud por desarrollar contenidos basados en este paradigma para aportar a sus estudiantes las oportunidades que permitan lograr aprendizajes automotivados. Pese a esto, aún no hay resultados concluyentes del impacto en el aprendizaje en Ciencias de la Salud, y los reportes han llegado a tener resultados contradictorios $(11,12)$.

A nivel de procesos de enseñanza-aprendizaje en ciclo previo a las clínicas de Medicina, es derrotero deseable el logro de aprendizaje significativo de las Ciencias Básicas en los estudiantes, pues se trata de conocimientos científicos fundamentales que pueden aplicarse en la resolución de problemas de los pacientes $(13,14)$. Existen experiencias del uso de videos instruccionales en temas fundamentales de Fisiología que posibilitan escenarios de clase invertida y transforman el aula en un espacio de reflexión, análisis y discusión de problemas a partir de instrucción brindada previamente a los estudiantes mediante estos videos $(15,16)$.

En la enseñanza de la Fisiología se han publicado algunas experiencias interesantes sobre el uso del webcast. Tune y cols, en un ejercicio de curso invertido, encontraron que los estudiantes que recibieron el módulo de cardio respiratorio por medio de videos tuvieron una diferencia porcentual de 12 puntos en los exámentes, por encima de los que asistieron presencialmente a las clases (16). Por su parte Rae y McCarthy utilizaron el webcast antes de las clases magistrales de Fisiología en un curso pre-médico, encontrando que este grupo obtuvo calificaciones significativamente más altas en varios formatos de examen en comparación con el grupo control, pero solo el 15\% de la clase informó haber revisado la totalidad del material entregado en este formato (17). No obstante estos mismos investigadores en otro estudio, encuestaron a 191 estudiantes, encontrando que el $79 \%$ de los estudiantes que recibieron los webcast previos a la clase magistral, manifestaron su preferencia por esta última estrategia de enseñanza(18). En nuestra región, Mejía y cols, encontraron que el 75\% de los estudiantes de un curso de Bioquímica expuestos a la metodología de caso clínico con uso de video, la consideraron como excelente estrategia para entender las respuestas fisiológicas en los casos analizados (19). El objetivo de esta investigación educativa es evaluar el impacto en el aprendizaje de temas de Fisiología cardiorrespiratoria mediante un webcast aplicado en estudiantes de Medicina de una universidad colombiana, cuyo ciclo preclínico (Ciencias básicas médicas), aborda de manera integrada el estudio de la estructura y función del organismo humano durante los tres primeros semestres, durante el cuarto semestre los procesos patológicos y en el quinto semestre los procesos farmacológicos. 


\section{Metodología}

Se realizó un estudio cuasi-experimental con los estudiantes matriculados en segundo año de medicina de una universidad del nororiente colombiano. La muestra fue seleccionada por conveniencia y se tuvo como criterios de inclusión, estar matriculados en el tercer semestre (se estudiaron dos cohortes) y aceptar participar en el estudio mediante consentimiento informado escrito. Esta investigación fue evaluada por el Comité de Ética Institucional bajo lo estipulado en la Resolución 8430 de 1993 y considerado sin riesgo para los participantes.

Los estudiantes fueron aleatorizados en dos grupos:

- Webcast (recibieron un enlace vía correo electrónico para acceder a los contenidos presentados en formato webcast). Se les brindó libertad para usar el material cuantas veces quisieran y estaban exentos de asistir a las clases magistrales programadas sobre el tema.

- Metodología tradicional (clases magistrales presenciales y programadas, con una disertación del tema por parte del docente).

El módulo de Fisiología cardiorespiratoria fue administrado a través de cinco clases teóricas, se usaron los mismos materiales audiovisuales y las competencias a alcanzar fueron las mismas independientemente del método de instrucción. De forma adicional, quienes recibieron webcast fueron convocados a dos sesiones de una hora para resolver dudas. Todos los estudiantes asistieron a 3 talleres para complementar los temas del módulo. Se aplicó una breve encuesta con datos demográficos a todo el grupo y una adicional indagando el uso de los materiales en el grupo de quienes fueron asignados al webcast; en esta segunda encuesta se indagó en pregunta cerrada por los dispositivos utilizados, frecuencia del uso, modo de tomar el webcast, y en pregunta abierta la satisfacción frente a la herramienta en términos de comodidad al usarla, los aspectos favorables que le destacan y si tuvo algún dificultad o problema para usarla.

Para el desarrollo de los webcast, se tuvo apoyo del departamento de educación virtual de la universidad y se produjeron los videos usando el programa CAMTASIA; asimismo, fueron tenidos en cuenta aspectos pedagógicos y técnicos en la producción audiovisual, se incluía un menú de navegación dentro del recurso educativo y el mismo fue embebido dentro de un Sistema de Administración del Conocimiento (LMS) administrado por la universidad. Posterior a la investigación, los materiales fueron dispuestos como Recursos Educativos Abiertos (REA).

Los resultados evaluados cuantitativamente como medias y desviaciones estándar sobre la calificación obtenida en la evaluación de Fisiología, utilizando el 3.0 como valor para aprobar, en una escala de 1 a 5 . Se realizó un análisis univariado ANOVA para comparaciones múltiples entre los grupos usando el programa STATA 8.0 y se tomó un valor significativo de error alfa en 0.1 y menores. Las medidas de dispersión se expresan en la media y el Error Estándar de la Media(EEM).

\section{Resultados}

Participaron 135 estudiantes, con una mayoría en el grupo que recibió los contenidos mediante la clase magistral, los detalles de la población estudiada se presentan en la Tabla 1. La diferencia entre estos dos grupos se debió a que algunos de ellos manifestaron su deseo de no hacer parte del grupo webcast una vez conocieron el resultado de la aleatorización $(n=7)$ y en consecuencia fueron asignados a la metodología tradicional.

Tabla 1. Composición del grupo de personas participantes del estudio.

\begin{tabular}{lcc}
\hline Participantes $(\mathrm{n}=135)$ & $\mathbf{n}$ & $\%$ \\
\hline Hombres & 51 & $38 \%$ \\
\hline Mujeres & 84 & $62 \%$ \\
\hline Grupo metodología tradicional & 74 & $55 \%$ \\
\hline Grupo metodología webcast & 61 & $45 \%$ \\
${ }^{*}$ n: número & & \\
\end{tabular}

Las características de la población de estudiantes en el programa de Medicina en donde se aplicó este estudio es similar a la de otros programas en Colombia en donde hay predominio de mujeres y las poblaciones universitarias son mayoritariamente jóvenes; la edad de la mayoría de participantes en el estudio osciló entre 16 y 20 años, excepto dos estudiantes que eran mayores de 35 años. Lo anterior es relevante para el contexto relacionado con el uso de las TIC.

En cuanto al uso de la herramienta webcast, como modalidad de instrucción, se encontraron los siguientes resultados: el dispositivo más utilizado fue el laptop o computadora portátil $(69 \%)$ seguida de la computadora de escritorio o desktop $(25.4 \%)$ y un bajo porcentaje hizo uso de dispositivos móviles para su revisión. Aproximadamente un $80 \%$ de los estudiantes revisaron los contenidos en más de una ocasión, y de éstos $18 \%$ manifestaron haberlo hecho cinco o más veces). La mayor parte de los participantes visualizaron los webcast de principio a fin $(70.4 \%)$ de forma ininterrumpida, un $39.4 \%$ los revisaron de forma completa pero por capítulos, usando saltos en los menús disponibles en la herramienta y $14.1 \%$ los revisaron por segmentos, enfocados en temas donde tenían dudas tras haber usado otros métodos de instrucción como la lectura de textos guía recomendados por los docentes. El $81.7 \%$ de los usuarios manifestó haberse sentido cómodo con el uso de la herramienta. Algunas de las razones por las cuales hubo insatisfacción con el uso de la herramienta tenían que ver con aspectos técnicos; casi la cuarta parte de los 
participantes de este grupo manifestó haber tenido algún tipo de dificultad técnica con la visualización de los contenidos, y se detectó que había incompatibilidades con dispositivos del sistema operativo iOS y MacOS los cuales fueron prontamente atendidos por el equipo de educación virtual de la universidad; sin embargo, pese a que se trató de resolver los problemas de adaptabilidad, no hubo adherencia al uso de dispositivos ligeros, sobre todo con las tabletas y teléfonos inteligentes posiblemente debido al hecho de que ya los participantes habían asegurado que les funcionaba en una computadora y decidieron no hacer pruebas ulteriores con otros dispositivos.

De otra parte, el 78.9\% de los participantes en el grupo webcast describieron los conceptos presentados como claros, integrales y amenos, un $16.9 \%$ mencionaron que la presentación fue poco amena pero los conceptos claros y completos, y un $4.2 \%$ indicaron que el material fue confuso pero ameno y completo.

Respecto a la medición del impacto en el aprendizaje de los estudiantes, se utilizó una prueba de selección múltiple con única mejor respuesta, pero a diferencia de la prueba utilizada tradicionalmente que muchas veces se fundamenta en la evocación de conceptos, se diseñaron preguntas que los implicará de mayor manera en el razonamiento crítico, la resolución de problemas, la proposición de hipótesis es decir que representara un mayor reto cognitivo para el estudiante y permitiera así la transferencia de aprendizajes significativos. Los resultados se evaluaron a partir de las calificaciones obtenidas en una escala de cero a cinco y con calificación aprobatoria igual o mayor a 3.0. El promedio obtenido en el grupo de metodología tradicional fue de 3.40 $( \pm 0.062, E E M)$ frente a $3.50( \pm 0.065, E E M)$ en el grupo de webcast (Figura 1); cuando se comparó la tasa de reprobación se encontró que esta fue menor en el grupo de webcast frente al grupo de metodología tradicional $(22.95 \%$ versus $25.68 \%$ respectivamente). Un análisis posterior dentro del grupo de reprobados encontró que hubo peor desemneño en el oruno de la clase tradicional comnarado

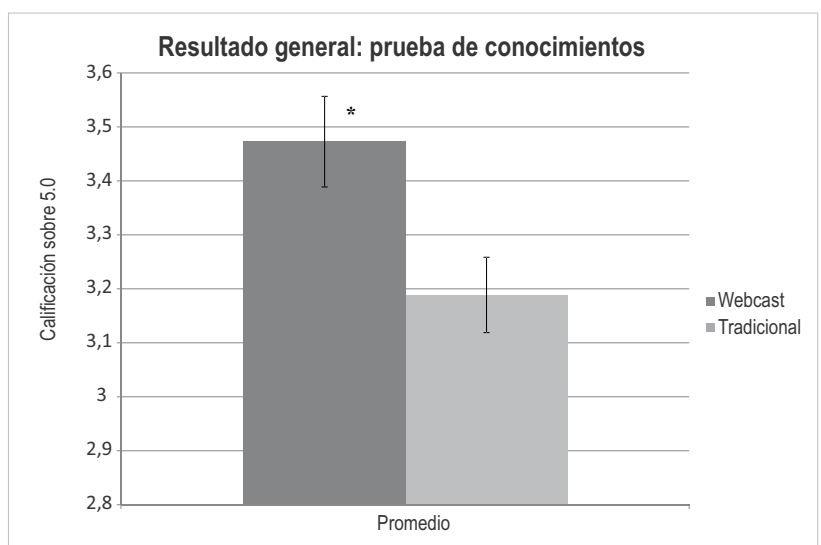

Fuente: Elaboración de los autores.

Figura 1. Calificación promedio de ambos grupos en la prueba escrita de conocimientos. con los que se instruyeron por webcast $(\mathrm{x}=2.72 \pm 0.051$ versus $2.86 \pm 0.039, \mathrm{EEM}$ ) respectivamente (ANOVA, F= $3.672, p=0.06$ ) (Figura 2). En el grupo de estudiantes que aprobaron la prueba de conocimientos no existieron diferencias significativas entre las metodologías aplicadas. El riesgo relativo para reprobar la evaluación general de conocimientos en una prueba de selección de única respuesta entre opciones múltiples fue 1.11 en el grupo de metodología tradicional (IC 95\% $=0.52-2.56$ ).

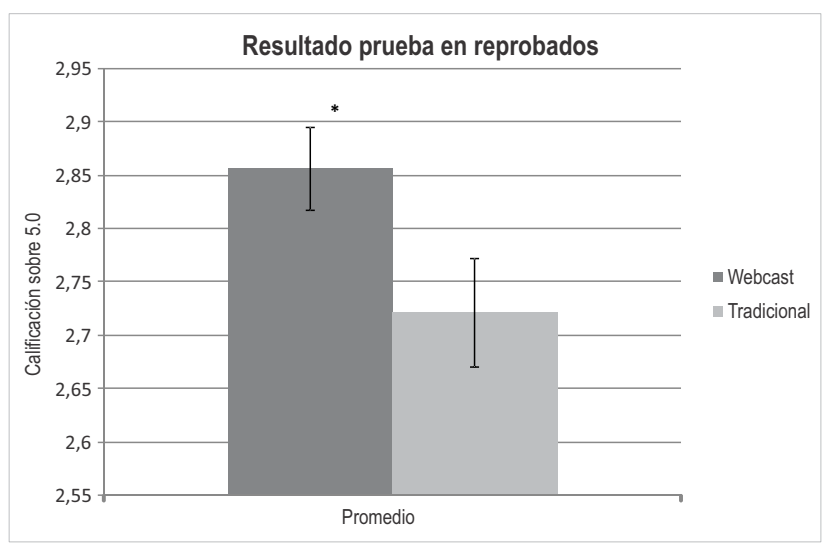

Fuente: Elaboración de los autores.

Figura 2. Calificación promedio de quienes reprobaron el examen de conocimientos según el método de instrucción. $\left({ }^{*}\right.$, ANOVA, $\left.\mathrm{F}=3.67, \mathrm{p}=0.06\right)$

Adicionalmente se hizo un análisis independiente sobre el desempeño en las preguntas de cada subsistema evaluado y se encontró un mejor resultado en las preguntas de fisiología cardiovascular en el grupo que recibió webcast al ser comparados con el grupo del método tradicional $(\mathrm{x}=3.47 \pm$ 0.04 versus $3.18 \pm 0.03, \pm E E M ; A N O V A, F=6.56, p=0.01$ ) (Figura 3); adicionalmente, hubo un riesgo aumentado para reprobar estas preguntas en el grupo de la clase tradicional $(\mathrm{R} R=1.42)$. No hubo diferencias entre grupos en las preguntas de fisiología respiratoria.

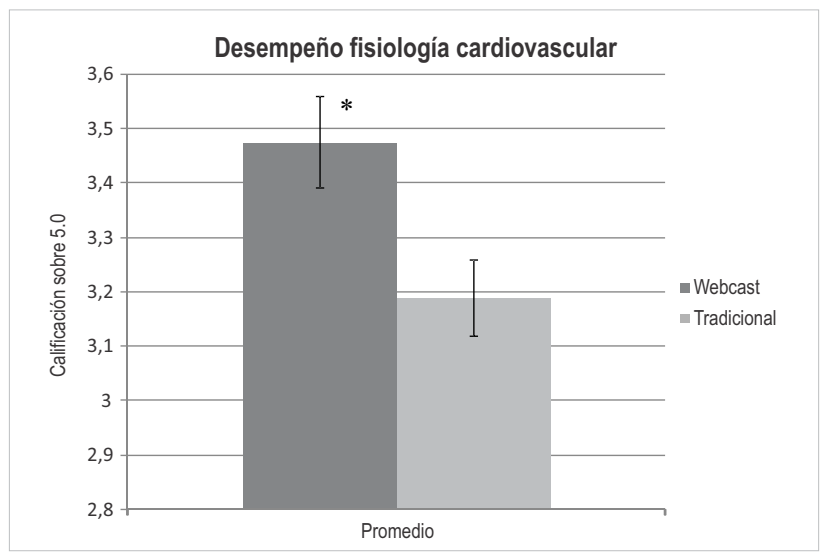

Fuente: Elaboración de los autores.

Figura 3. Calificación obtenida en la prueba de conocimientos concerniente al componente fisiología cardiovascular. $\left({ }^{*}\right.$, ANOVA, $\left.F=6.56, p=0.01\right)$ 


\section{Discusión}

Los métodos tradicionales de enseñanza de la medicina a nivel mundial se han fundamentado en un rol pasivo del aprendiz (20), por lo que lo que los avances en pedagogía sumado al desarrollo de las TIC se han orientado a que el estudiante asuma un papel más protagónico en su propio proceso de aprendizaje, que este perdure en el tiempo y pueda ser utilizado y transferido en la resolución de los problemas que se le puedan presentar en el ejercicio profesional, es decir, a que se logren aprendizajes significativos. Si bien en este estudio los resultados en las evaluaciones no mostraron diferencias significativas entre los dos grupos, cabe resaltar que la enseñanza de los contenidos a través de webcast, tampoco generó un perjuicio, al ser comparado con las clases magistrales. Lo anterior, debido a que existe la creencia de que los estudiantes, y más aún si son muy jóvenes, no tienen la preparación para asumir responsabilidades mayores en su proceso de enseñanza - aprendizaje que implican estos métodos fundamentados en la autonomía, además de que se les considera como propiciadoras de escaso esfuerzo, procrastinación o, desde otro ángulo, que el estudiante universitario aún necesita de la presencia obligada del docente dentro de un recinto académico para que pueda adquirir el conocimiento (21). Los resultados de esta investigación sobre una innovación educativa van en consonancia con lo descrito por un grupo chileno de profesores de Medicina: "se puede afirmar que la falta de clases no produjo un deterioro en los conocimientos evaluados por estas pruebas"(22).

Otro hallazgo interesante es que los estudiantes primordialmente usaron computadoras portátiles seguidas por computadoras de escritorio para acceder a los contenidos de webcast, sin que hubiera un franco uso intensivo de dispositivos móviles, lo que indica que este tipo de estudiantes, aunque nativos digitales, no están inmersos en el m-learning.

Pese a que una de las limitaciones del presente estudio es que el tamaño de muestra es pequeño en relación con lo medido como desenlace en el cuestionario de evaluación (calificación mayor a 3.0); sin embargo, parece indiciarse que el uso de webcast podría brindar facilidades a los estudiantes que les permitirían reducir su riesgo de reprobación en dicha prueba, debido a factores como la posibilidad de revisar varias veces las grabaciones, complementar con otras fuentes de información o ahondar en conocimientos al poder realizar búsquedas en Internet mientras se accede al webcast y estos factores podrían favorecer el aprendizaje significativo, hallazgos que tienen similitud con otros estudios en donde se halló un mejor rendimiento en estudiantes de postgrado médico que habían recibido instrucción por webcast comparado con los que habían recibido las clases magistrales tradicionales (23). Asimismo, la repetición de la información es una estrategia para fortalecer la memoria a largo plazo desde donde podrá evocarse la información con mayor facilidad; sin embargo, la emoción también entra a jugar un rol importante en estos procesos de consolidación y evocación ya que un material poco atractivo reduce el interés del estudiante y crea interferencia en estos procesos de memoria explícita asociados al hipocampo y otras estructuras del circuito de Papez implicados en la resolución de un cuestionario de opción con única respuesta correcta a partir de múltiples opciones y que no participan en la demostración de una competencia procedimental en donde los sustratos anatómicos de la memoria están relacionados con el cerebelo y los núcleos basales(24).

Respecto a los resultados sobre fisiología cardiovascular, es importante tener en cuenta que los participantes ya habían tenido contacto con este tema en un módulo previo; no obstante, al revisar el desempeño en las preguntas de esta parte de la fisiología, el grupo de webcast tuvo mejores resultados y que posiblemente esta estrategia aporta en el aprendizaje significativo de temas previamente abordados; es evidente que este aspecto no fue una hipótesis inicial de esta investigación y que se requeriría de estudios futuros dirigidos específicamente a este objetivo para dilucidar este hallazgo.

La construcción de los webcast demandó un esfuerzo importante por la preparación de los materiales en cuanto a la selección de contenidos, organización de ayudas en imágenes, requerimientos pedagógicos, grabación y empaquetamiento en un contenedor multimedia. En general hubo buena aceptación del producto en los estudiantes y es importante tener en cuenta que esta metodología puede favorecer en mayor grado a quienes tienen estilos de aprendizaje predominantes en los rasgos visual y auditivo, aspecto que no fue evaluado en este artículo. Adicionalmente, el desarrollo de un material amigable con aquellos considerados como "nativos digitales" basado en tecnologías de web 2.0 y con el respaldo científico y pedagógico de una institución de educación superior y disponible como un REA, alienta a los estudiantes a preferir este tipo de fuentes de información sobre otras poco profesionales que en ocasiones corresponde a los primeros resultados de los motores de búsqueda más populares $(25,26)$.

En virtud de lo anteriormente expuesto, es una necesidad la adquisición de competencias profesionales en el manejo de información y exploración de datos para seleccionar fuentes confiables y descartar las imprecisas y malintencionadamente erróneas (27), debido al fenómeno de replicación de información que ocurre actualmente en la sociedad de la información y la popularización de sitios en donde los usuarios pueden publicar información fácilmente y sin pasar por un proceso de revisión y arbitraje; instituciones como la Federación Americana de Editores reportan la publicación diaria de casi 85,000 artículos de diversos orígenes relacionados con Ciencias de la Salud y las revistas científicas especializadas emiten a diario alrededor de 1,000 
artículos nuevos (28). Se estima que cada lustro, se renueva cerca de 75\% del conocimiento médico (29) y otros autores como Burwell dan un plazo de un decenio para que un concepto actual en Medicina llegue a revaluarse por equívoco(30).

\section{Conclusiones}

El objetivo fundamental de lograr el aprendizaje significativo cobra relevancia a la hora de formar los estudiantes; en este estudio, un porcentaje significativo de quienes recibieron webcast como método de instrucción se sintió satisfecho con los conocimientos adquiridos ya que les permitió desempeñarse de forma favorable en las pruebas de conocimientos sobre fisiología cardio-respiratoria; no obstante, debe tenerse en cuenta que la clase magistral aún tiene una importante relevancia en la formación universitaria al brindar ventajas como la posibilidad de resolver dudas en el momento que surgen, gozar del carisma de los docentes con sus conocimientos amplios y robustos sobre el tema, entre otros, que también permiten adquirir los conocimientos para el desempeño de la profesión.

En conjunto, la experiencia de investigación educativa del presente estudio nos indica que, para la formación de profesionales en Ciencias de la Salud, posiblemente sea la mejor estrategia el construir currículos con clases magistrales apoyadas en webcast seleccionados que puedan revisarse antes de asistir al encuentro presencial con el docente. Crear este tipo de programas, seguramente requiere reorganizarse el tiempo y actividades de trabajo en el aula, posibilitando el desarrollo de estrategias como el aprendizaje basado en proyectos o modificaciones del aprendizaje basado en problemas, que estimulen procesos formales del pensamiento y propendan por la adquisición de competencias más relacionadas con la parte superior de la pirámide de Miller(31).

Uno de los aspectos a resolver con el uso de TIC en la educación superior actualmente es que en mayoría los estudiantes son nativos digitales mientras que los docentes son migrantes digitales (26), esto crea una brecha fundamentada en el escepticismo y temor de los últimos sobre la aplicación de las TIC en el logro de las compentencias deseadas en los estudiantes, requiriéndose así el concurso de equipos interdisciplinarios para el desarrollo de los recursos educativos para lograr productos con excelente contenido, pedagogía y adaptabilidad a diferentes plataformas tecnológicas, de forma tal que sea posible albergar procesos bien planificados de enseñanza aprendizaje que permitan a los estudiantes adquirir las habilidades necesarias para un buen desempeño profesional. Otro aspecto importante a resolver es que el desarrollo de los REA demanda una inversión de tiempo y dinero que las instituciones educativas deben estar dispuestas a asumir, ya que en muchas ocasiones se requiere el uso de aplicaciones informáticas que cobran por sus licencias, junto a los docentes que necesitan tiempo laboral para planear, ejecutar, hacer pruebas y ajustes, asesorar en el uso del recurso a quienes así lo necesiten y finalmente, tener un proceso de retroalimentación con sus pares y los estudiantes que les permita mejorar lo construido para optimizar los resultados(28).

\section{Responsabilidades éticas}

Protección de personas y animales. Los autores declaran que los procedimientos seguidos se conformaron a las normas éticas del comité responsable y de acuerdo con la Asociación Médica Mundial y la Declaración de Helsinki.

Confidencialidad de los datos. Los autores declaran que han seguido los protocolos de su centro de trabajo sobre la publicación de datos de pacientes.

Derecho a la privacidad y consentimiento informado. Los autores han obtenido el consentimiento de los pacientes y/o sujetos referidos. Este documento obra en poder del autor de correspondencia.

\section{Conflicto de intereses}

Los autores declaran no tener conflicto de interés.

\section{Financiación}

El presente proyecto de investigación fue financiado en su totalidad por la Universidad Autónoma de Bucaramanga, proyecto 134071 de convocatoria interna.

\section{Referencias}

1. Salinas J. Modelos emergentes en entornos virtuales de aprendizaje. [Internet]. Congr Int Edutec. 2009. [citado el $28 \mathrm{de}$ mayo 2017]. Disponible en: http://gte.uib.es/pape/gte/content/modelos-emergentesen-entornos-virtuales-deaprendizaje

2. García $H$, Navarro L, López $M$, Rodríguez $M$. Tecnologías de la Información y la Comunicación en salud y educación médica. Rev EDUMECENTRO. 2014; 6(1):1-9

3. Cabero J. Reflexiones educativas sobre las tecnologías de la información y la comunicación (TIC). Rev Tecnol Cienc y Educ. 2015; 1(1):19-27.

4. Bates A. Teaching in a digital age [Internet]. Vol. 1, Open Web Source. 2015. 501 p. Disponible en: http://opentextbc.ca/teachinginadigitalage/

5. Badillo R. Aplicaciones y Estrategias "Web 2.0" en la Educación Médica. Revista Científica Salud Uninorte. $2011 ; 27(2): 275-88$. 
6. Masic I. E-learning as new method of medical education. Acta Inform Med. 2008; 16(2):102-17. doi: 10.5455/aim.2008.16.102-117

7. JAMA Editors. JAMA Videos [Internet]. United States; 2017 [citado el 28 de mayo de 2017]. Disponible en: https://www.youtube.com/user/JAMAJournalVideo

8. NEJM editors. NEJMvideo [Internet]. United States; 2017 [citado el 28 de mayo de 2017]. Disponible en: http://www.nejm.org/multimedia/medical-videos

9. JHM Marketing \& Communications. Johns Hopkins Medicine [Internet]. United States; 2017. [citado el 28 de mayo de 2017]. Disponible en: https://www. youtube.com/user/JohnsHopkinsMedicine

10. Masters K, Ellaway R, Topps D, Archibald D, Hogue R. Mobile technologies in medical education: AMEE Guide No. 105. Med Teach. 2016; 38(6):537-49. Disponible en: https://doi.org/10.3109/0142159X.2016.1141190

11. Schreiber B, Fukuta J, Gordon F. Live lecture versus video podcast in undergraduate medical education: $A$ randomised controlled trial. BMC Med Educ. 2010; 10(1):68. Disponible en: https://doi.org/10.1186/14726920-10-68

12. McNulty J, Hoyt A, Gruener G, Chandrasekhar A, Espiritu B, Price R, et al. An analysis of lecture video utilization in undergraduate medical education: associations with performance in the courses. BMC Med Educ. $2009 ; 9(1): 6$. Disponible en: https://doi.org/10.1186/1472-6920-9-6

13. Michael J. In pursuit of meaningful learning. Adv Physiol Educ. 2001; 25(3):145-58.

14. Gonzalez H, Palencia A, Umaña L, Galindo L, Villafrade L. Mediated learning experience and concept maps: a pedagogical tool for achieving meaningful learning in medical physiology students. AJP Adv Physiol Educ. 2008; 32(4):312-6. Disponible en: https://doi.org/ 10.1152/advan.00021.2007

15. Gilboy M, Heinerichs S, Pazzaglia G. Enhancing student engagement using the flipped classroom. J Nutr Educ Behav. 2015; 47(1):109-14. Disponible en: https://doi.org/10.1016/j.jneb.2014.08.008

16. Tune J, Sturek M, Basile D. Flipped classroom model improves graduate student performance in cardiovascular, respiratory, and renal physiology. AJP Adv Physiol Educ. 2013; 37(4):316-20. Disponible en: https://doi.org/10.1152/advan.00091.2013

17. Rae M, McCarthy M. The Impact of Vodcast Utilisation upon Student Learning of Physiology by First Year Graduate to Entry Medicine Students. J Scholarsh Teach Learn. 2017; 17(2):1-23. Disponible en: https://doi.org/10.14434/josotl.v17i2.21125

18. Rae M, O'Malley D. Do prerecorded lecture VODcasts affect lecture attendance of first-yearpre-clinical Graduate Entry to Medicine students? Med Teach. 2017; 39(3):250-4. Disponible en: https://doi.org/
10.1080/0142159X.2017.1270436

19. Mejía Ó, García A, García G. Técnicas didácticas: método de caso clínico con la utilización de video como herramienta de apoyo en la enseñanza de la medicina. 2015;45(2):29-38.

20. National Geographic. La medicina en la Grecia antigua: el nacimiento de una ciencia [Internet]. Historia NG. 2013 [citado el 19 de agosto de 2017]. Disponible en: http://www.nationalgeographic.com.es/historia/grandes -reportajes/la-medicina-en-la-grecia-antigua_7023/2

21. Guiraldes H, Oddó H, Mena B, Velasco N, Paulos J. Enseñanza de la anatomía humana: experiencias y desafíos en una escuela de medicina. Rev Chil anat. 2001; 19(2):205-12. Disponible en: http://dx.doi.org/ $10.4067 /$ S0716-98682001000200013

22. Moreno R, Cruz E, Lisboa C, Pertuzé J. Modificaciones en la enseñanza de enfermedades respiratorias. Bol. Esc. Med. 1997; 26(3):112-5.

23. Andolsek K, Murphy G, Pinheiro S, Petrusa E, Tuck T, Weinerth J. Efficacy and efficiency of webcast orientations versus live resident orientations: results of a 2-year survey. J Grad Med Educ. 2010; 2(1):136-40. Disponible en: https://doi.org/10.4300/JGME-D-0900105.1

24. Delgado H. El triple componente cromático de las experiencias: memoria, emoción y sistema nervioso autónomo. MedUNAB. 2009;12(1):52-61.

25. García F, Portillo J, Romo J, Benito M. Nativos digitales y modelos de aprendizaje. [Internet]. [citado el 20 de abri d e $\left.\quad \begin{array}{llll}2 & 0 & 1 & 7\end{array}\right]$; $\quad$ D i s p o n i b le e n : https://www.businessintelligence.info/assets/varios/nati vos-digitales.pdf

26. Prensky M. Digital Natives, Digital Immigrants Part 1. On the Horizon. 2001; 9(5):1-6. Disponible en: https://doi.org/10.1108/10748120110424816

27. Granados A. La sanidad en la era de la sociedad de la información [Internet]. Boletín de la Agencia de Evaluación de Tecnología. 1999 [citado el 27 de marzo de 2017]. Disponible en: https://goo.gl/7Exqym

28. González T. Nuevas tendencias en los sitios web de salud y medicina. Hipertext.net [Internet]. 2006 [citado el 24 de marzo de 2017];4(1). Disponible en: http://ddd.uab.cat/pub/artpub/2003/88756/hipertext_a2 003n1a11/salud.html

29. Isaza A. Clases magistrales versus actividades participativas en el pregrado de medicina. De la teoría a la evidencia. Rev Estud Soc. 2005; (20):83-91.

30. Giuse N. Riding the waves of change together: are we all paying attention? J Med Libr Assoc. 2008; 96(2):85-87. Disponible en: https://doi.org/10.3163/15365050.96.2.85

31. Miller G. The Assessment of Clinical Skills/Competence/Performance. Acad Med. 1990; 65(9):S63-7. 